\title{
Analyse multidisciplinaire du processus décisionnel de la CISR
}

\author{
François Crépeau, Patricia Foxen, France Houle et Cécile Rousseau
}

\section{Résumé}

La détermination du statut de réfugié est l'une des tâches décisionnelles les plus difficiles à accomplir dans notre société. Les décideurs qui sont engagés dans ce processus doivent posséder une connaissance adéquate du contexte culturel, social et politique du pays d'origine, avoir la capacité de supporter le fardeau psychologique de l'audience de cas difficiles et de décisions pouvant mettre des vies en danger, et être aptes à examiner des questions juridiques et des éléments de preuve de grande complexité. Au Canada, en dépit d'un taux d'acceptation relativement élevé et d'une interprétation large de la définition internationale des réfugiés, beaucoup d'acteurs engagés dans le processus de détermination ainsi que le public en général ont exprimé leur insatisfaction à la Commission de l'immigration et $d u$ statut de réfugié (CISR) quant au mécanisme décisionnel. Le rapport qui suit traite de la nature et de la cause des désaccords qui existent entre les différents acteurs engagés dans le processus, dont font partie d'un côté des membres de la Commission de la CISR et des agents d'évaluation, et de l'autre des professionnels tels que avocats, médecins et témoins experts. Faisant appel à une structure méthodologique qui comprend des approches tant qualitatives que quantitatives, les auteurs de ce projet de recherche pluridisciplinaire ont défini un groupe de paramètres à l'aide desquels ils ont examiné trois dimensions critiques $d u$ processus de détermination du statut de réfugié, soit les dimensions culturelles, psychologiques et juridiques. Les données utilisées dans le cadre de cette étude touchent quarante cas problématiques rapportés à l'équipe de recherche par des avocats et d'autres professionnels: dix de ces cas, documentés par des cassettes et des transcriptions d'audiences, furent analysés en profondeur par des experts appartenant aux trois champs d'études respectifs. Les résultats indiquent que le processus décisionnel est affecté par un grand nombre de problèmes liés à des facteurs culturels (connaissance inadéquate de la situation politique dans les pays d'origine, représentations erronées de la vie quotidienne dans les pays en guerre et stéréotypes culturels ou préjugés), psychologiques (difficultés à pouvoir réagir sainement aux traumatismes vicariants et aux réactions émotionnelles) et juridiques (difficultés à évaluer les preuves, à évaluer la crédibilité, à conduire des audiences et à rédiger des décisions). Dans la majorité des cas, ces dimensions culturelles, psychologiques et juridiques interagissent entre elles, renforçant l'incertitude, le cynisme et l'agressivité, et générant des répercussions négatives sur la capacité des membres de la Commission à évaluer correctement la crédibilité et sur le bon déroulement des audiences en général. Le rapport renferme une série de recommandations, dont l'amélioration de la sélection des commissaires et des agents d'audience, ainsi que des programmes de formation et de soutien pour tous les acteurs. Il met aussi en lumière les difficultés inhérentes à un système qui fait usage d'un processus non contradictoire dans des audiences pour déterminer le statut de réfugié. Une version anglaise de ce rapport se trouve à l'adresse suivante: http://www .juris.uqam.ca/cedim/recherches.htm.
Abstract
The refugee determination process is one of the most complex judicial functions in our societies. Decision makers involved in this process require a sufficient knowledge of the cultural, social, and political environment of the country of origin, a capacity to bear the psychological weight of difficult hearings and potentially life-threatening decisions, and an ability to deal with complex legal issues and evi- dence. In Canada, despite a relatively broad recognition rate and wide interpretation of the international refugee definition, dissatisfaction with the decision-making process 
at the Immigration and Refugee Board (IRB) has been expressed by numerous actors as well as by the general public. The following report examines the nature and causes of discord between the different actors involved in this process, including IRB board members and refugeeclaim officers on the one hand, and professionals such as lawyers, doctors, and expert witnesses on the other. Using a methodological framework that includes both quantitative and qualitative approaches, the authors of this multidisciplinary research project define a set of parameters and variables through which three critical dimensions of the refugee determination process - cultural, psychological, and legal - are explored. Data for the study consist of forty problematic cases referred to the research team by lawyers and other professionals; ten of the cases, which include cassettes and transcripts of hearings, were analyzed in depth by experts in the three respective fields of study. The results indicate numerous problems affecting decisionmaking based on legal factors (difficulties in evaluating evidence, assessing credibility, conducting hearings, and writing decisions), psychological factors (difficulties in coping with vicarious traumatization and emotional reactions), and cultural factors (poor knowledge of the political situation in countries of origin, false representations of daily life in war-torn countries, and cultural misunderstandings or insensitivity). In a majority of cases, these legal, psychological, and cultural dimensions interact, reinforcing uncertainty, cynicism, and aggression, and often negatively affecting the ability of board members to evaluate credibility and the overall conduct of hearings. The report proposes a set of recommendations including revised selection criteria for board members and refugee claim officers, as well as improved training and support for all actors; it highlights as well the difficulties inherent in using a non-adversarial system in refugee hearings. An English version of the report can be found at http://www.juris .uqam.ca/cedim/recherches.htm.

\section{Problématique du projet}

$1 \begin{aligned} & \text { u cours des onze dernières années, le processus de } \\ & \text { reconnaissance du statut de réfugié canadien a été } \\ & \text { critiqué par nombre de ses acteurs. On dénonce }\end{aligned}$ l'insouciance de certains avocats, l'incompétence de certains commissaires de la Commission de l'immigration et du statut de réfugié ${ }^{1}$ l'agressivité de certains agents d'audience, la partialité de certains interprètes, etc.

Beaucoup croient que la cISR est victime de demandeurs d'asile et d'avocats astucieux. Un fonctionnaire du minis- tère de la Citoyenneté et de l'Immigration est allé jusqu'à dire que seulement $10 \%$ des demandeurs avaient besoin de protection et que seulement $1 \%$ d'entre eux nécessitaient la protection du Canada, ce qui équivaut à cinquante fois moins que le taux d'acceptation moyen de la cisR. Voilà la piètre opinion que certains fonctionnaires peuvent avoir de l'efficacité de la cisr. D’autres personnes estiment que, dans bien des cas, les réfugiés ne sont pas traités équitablement et que des commissaires, incompétents et particulièrement insensibles au sort des demandeurs, ne prêtent souvent que peu d'attention aux risques de torture ou de mauvais traitements en cas de renvoi, ce qui se traduit par de véritables « histoires d'horreur».

Nous ne pouvons croire d'emblée ces allégations, ne serait-ce que parce qu'elles se contredisent. Mais il existe un sentiment palpable de méfiance envers les résultats du processus. Nous voulions savoir pourquoi.

Nous croyons que la détermination du statut de réfugié est l'une des tâches décisionnelles les plus difficiles à accomplir dans notre société. Nous croyons également que la majorité des acteurs travaillent de bonne foi et avec beaucoup de bonne volonté. Notre but n'est pas de discréditer la CISR auprès des ONG, de la communauté juridique, du Ministère ou des médias, comme certains le souhaiteraient.

En 1998, la ministre de la Citoyenneté et de l'Immigration a tenu des consultations publiques en vue de modifier la Loi sur l'Immigration. À la suite de l'invitation de la ministre à participer à ces consultations, les professeurs François Crépeau et France Houle avaient présenté un mémoire dans lequel ils recommandaient notamment de resserrer les critères de sélection des commissaires. On y proposait une liste de connaissances et d'habiletés de base qui nous apparaissaient essentielles pour garantir la compétence de ces dermiers². Cette préoccupation était absente de l'esprit du législateur lorsque, il y a onze ans, il a créé la cisR. Sans qu'une réflexion ait été amorcée sur cette question, le législateur a tout simplement institué un tribunal composé de personnes sans formation particulière. Ce choix, si tant est qu'il y en ait eu un, n'est pas répréhensible en soi, puisque la fonction prédominante des commissaires est d'évaluer la crédibilité des témoins. À la manière d'un jury, les commissaires prennent des décisions fondées sur le sens commun pour tirer des conclusions sur la crédibilité du demandeur de statut. Toutefois, il est problématique de postuler l'existence d'un "sens commun ", surtout en matière de détermination du statut de réfugié. Ce postulat est contestable sur deux plans : culturel et psychologique.

D'un point de vue culturel, il est erroné de prétendre que le point d'ancrage de ce «sens commun » correspond

(C) François Crépeau, Patricia Foxen, France Houle et Cécile Rousseau, 2001. Cette œuvre en libre accès fait l'objet d'une licence Creative Commons AttributionNonCommercial 4.0 International License, laquelle autorise l'utilisation, la reproduction et la distribution de l'œuvre sur tout support à des fins non commerciales, pourvu que l'auteur ou les auteurs originaux soient mentionnés et que la publication originale dans Refuge : revue canadienne sur les réfugiés soit citée. 
aux représentations et aux normes culturelles des Canadiens. Les demandeurs de statut témoignent d'événements qui comportent peu sinon aucune similarité avec la vie quotidienne des décideurs. Bien souvent ces derniers sont nés au Canada et, même s'ils ont émigré au Canada, ils sont rarement de la même nationalité ou du même groupe ethnique que les demandeurs dont ils doivent écouter les récits. Or, les recherches montrent que les individus de cultures différentes éprouvent plus de difficulté à transmettre et à comprendre les renseignements qu'ils échangent ${ }^{3}$. Outre la barrière linguistique qui est résolue par le moyen d'interprètes à la $\mathrm{CISR}^{4}$, il faut également mentionner la difficulté potentielle de comprendre la signification du langage non verbal de l'autre5. Les différences culturelles se meuvent aussi dans des univers de représentations mutuelles où les préjugés, les stéréotypes et le racisme implicite ou explicite peuvent être présents ${ }^{6}$. Finalement, les contextes sociopolitiques dans lesquels s'insèrent les histoires des demandeurs sont souvent complexes et nuancés, et ne peuvent être transmis dans un cadre narratif cohérent et structuré. La mise en évidence de toutes ces représentations culturelles est très importante, étant donné que celles-ci structurent la compréhension des décideurs du récit du demandeur de statut de réfugié.

Sur le plan psychologique, il faut mentionner deux phénomènes distincts. D'une part, les récits des demandeurs impliquent généralement des tortures, des viols, des détentions arbitraires, des menaces, des attaques armées. Ces événements peuvent engendrer des réactions psychologiques posttraumatiques chez les demandeurs, affectant la qualité de leur témoignage (la difficulté de se remémorer des détails tels que les dates, la description de lieux et le contenu de conversations, ou encore la difficulté de répondre directement et calmement aux questions). D'autre part, le récit des événements peut entraîner une transmission indirecte des traumatismes du demandeur au décideur? Ce dernier peut, pour se protéger, ériger des défenses menant, par exemple, au déni, à l'évitement, à la minimalisation de la gravité de situations, à des réactions émotives incontrôlées comme la colère, le manque d'empathie ou le cynisme. Ces réactions, conséquences directes de l'exposition massive à des récits traumatiques, influent directement sur la perception des décideurs de la crédibilité du demandeur ${ }^{8}$.

Par ailleurs, le processus comporte aussi des enjeux purement juridiques qui interagissent avec les dimensions culturelles et psychologiques. Le décideur ne peut pas accomplir sa tâche de manière isolée, il doit pouvoir intégrer son évaluation dans un cadre normatif complexe. En effet, le processus de détermination du statut de réfugié implique divers ordres juridiques. Sur le plan substantiel, le dé- cideur doit posséder des connaissances sur le droit international des réfugiés, le droit canadien de l'immigration et, dans une moindre mesure, le droit du pays d'origine du réfugié. Sur le plan formel, le décideur doit être à même d'appliquer les règles de preuve et de procédure devant les tribunaux administratifs. Il doit notamment veiller au déroulement efficace de l'audience, traiter équitablement la preuve, en donnant la possibilité au demandeur de réfuter tout renseignement qui lui est préjudiciable, et rédiger des motifs rationnels et convaincants de sa décision.

Afin d'examiner si les allégations d'incompétence des divers acteurs du processus sont fondées, nous avons constitué une équipe de recherche multidisciplinaire pour définir les problèmes juridiques, psychologiques et culturels qui alimentent le sentiment de méfiance. Sœur Denise Laîné, du CSAI, a réuni les auteurs de ce rapport pour lancer un projet pilote sur le processus de reconnaissance du statut de réfugié, dans le but de faire une évaluation préliminaire des rôles et des compétences de ses acteurs. Nous connaissions chacun le processus, soit pour l'avoir étudié, soit pour y avoir participé à l'occasion. Nous avons formé une équipe pluridisciplinaire de professionnels de la santé, de juristes et d'anthropologues, certains professeurs dans trois universités montréalaises (McGill, Université de Montréal, UQAM), des étudiants gradués avec une importante expérience de terrain, des professionnels traitant quotidiennement avec des étrangers, y compris des demandeurs d'asile.

\section{Cadre conceptuel du projet}

L'objectif général du projet de recherche était de découvrir la nature et les causes des désaccords existant, d'une part, entre des décideurs indépendants et impartiaux spécialisés, comme les commissaires ou des auxiliaires de justice tels les agents d'audience, et, d'autre part, des professionnels experts dans leur champ de pratique comme les avocats, les médecins et les psychologues. De façon plus particulière, nous voulions analyser le processus décisionnel en nous concentrant sur les facteurs culturels, psychologiques et juridiques qui influent sur les différents acteurs. Nous voulions suggérer des solutions effectives, allant au-delà des aménagements ad hoc qui sont souvent adoptés lorsque les médias révèlent une situation embarrassante. Notre objectif n'était pas de mesurer l'ampleur du phénomène sur une base quantitative, mais de faire une étude préliminaire et qualitative, qui rendrait possible une étude subséquente de la prévalence de ces problèmes.

Nous avons limité notre étude à des dossiers où des décisions ont été rendues par des commissaires du bureau de Montréal. Quatre-vingt-quatre dossiers nous ont été trans- 
mis par des avocats (6o \%) et par des intervenants professionnels et communautaires (40\%). À ce stade, nous avons demandé aux autorités de la CISR de s'associer à nos travaux, comme partenaire clé. Nous leur avons demandé de participer à la conception du cadre conceptuel et méthodologique de la recherche et de fournir des dossiers dans lesquels, à leur avis, certains acteurs auraient pu avoir un comportement problématique. La présidente d'alors a décliné notre invitation. Pour diverses raisons (impossibilité de contacter le réfugié pour obtenir un consentement, refus de celui-ci, absence de documents importants, etc.), nous avons exclu quarante-quatre dossiers, pour n'en conserver que quarante. Un profil sociodémographique de ces quarante dossiers est disponible, mais ne sera pas exposé en détail ici. Notons seulement que $87,5 \%$ de l'échantillon est arrivé au Canada entre 1995 et 1998. Ces dossiers ont été préalablement considérés par les professionnels impliqués comme problématiques relativement à deux critères. Le premier critère est que la demande de statut de réfugié a été refusée parce que le témoignage du demandeur a été jugé non crédible. Le deuxième critère est que l'évaluation de la crédibilité qui a été faite par les commissaires dans leurs décisions écrites révélait des manques importants sur le plan d'au moins une des catégories de facteurs étudiés : juridiques, psychologiques et culturels.

Dans un premier temps, nous avons établi les facteurs caractéristiques du rôle des acteurs, susceptibles d'expliquer les divergences dans leur perception et leur évaluation. La liste a été validée et complétée lors de l'analyse qualitative. Nous les avons divisés en trois catégories :

\section{Facteurs juridiques}

- Pour tous les acteurs, les difficultés à travailler dans le cadre d'une procédure de nature inquisitoire.

- Pour les commissaires et les agents d'audience, les difficultés à évaluer la recevabilité (preuve d'expert, preuve documentaire, etc.) et le poids de la preuve (l'importance des contradictions entre la Fiche de renseignements personnels et le témoignage oral des demandeurs; l'utilisation de la connaissance d'office; la compréhension de la situation sociale et politique dans le pays d'origine du demandeur; etc.).

- Pour les commissaires, les difficultés relatives à la conduite d'une audience (l'interrogatoire des demandeurs, le respect de la déontologie et de l'étiquette qui sied lors d'un processus décisionnel menant à la détermination de demandes de statut de réfugié).

- Pour les commissaires, les difficultés à rédiger les motifs de la décision.
- Pour les avocats représentant les demandeurs, les manques de préparation pour l'audience.

- Pour les interprètes, les difficultés à traduire correctement le témoignage du demandeur à cause des barrières interculturelles, comme les dialectes, le comportement verbal et non verbal, etc.

\section{Facteurs psychologiques}

- Pour tous les acteurs, mais tout spécialement pour les commissaires et les agents d'audience, les difficultés à transiger avec la transmission traumatique, telles que :

- les réactions massives d'évitement (évitement direct, déni, normalisation de situations extrêmes, pouvant prendre la forme de cynisme, etc.);

- les réactions émotives incontrôlées lors d'une confrontation à des expériences traumatisantes (colère, victimes perçues comme les agresseurs, manque d'empathie, formation réactionnelle, etc.);

- le manque de connaissance sur les conséquences psychologiques des traumatismes (symptôme et effets à court, à moyen et à long termes; relation victime/bourreau; etc.);

- l'utilisation inadéquate des rapports d'experts médecins et de psychologues (préjugés ou ignorance, statut de l'expert, tendance à prendre la place de l'expert, etc.).

- Pour les avocats, une tendance à exposer leur client à une retraumatisation, de manière à présenter un témoignage plus convaincant.

- Pour les professionnels de la santé :

- la tendance à prédire le comportement du demandeur devant le tribunal, sur le fondement des entrevues thérapeutiques (même si l'audition devant le tribunal est un environnement de survie radicalement différent de la relation sécurisante entre le patient et le professionnel);

- le manque de clarté dans l'évaluation :l'évaluation de la capacité de témoigner n'est souvent pas distinguée de l'évaluation du récit du patient comme explication des symptômes.

\section{Facteurs culturels}

- Pour tous les acteurs, mais tout spécialement pour les commissaires et les agents d'audience :

- le manque de connaissances sur la situation politique et sociale dans les pays d'origine;

- les représentations erronées de la vie quotidienne dans un pays en guerre ou en conflit; 
- les représentations simplistes de la cohérence sociale (stéréotypes);

- les malentendus culturels, l'insensibilité et les préjugés fondés sur le sexe, l'origine ethnique, la religion et l'orientation sexuelle;

- l'incompréhension des problèmes de temporalité et de cohérence narrative dans les récits de guerre et de fuite.

La détermination des facteurs adéquats a été une tâche continue, fondée sur les connaissances pratiques multidisciplinaires des chercheurs de l'équipe, puisque l'objectif visé était de mettre au point des outils de mesure (par la validation des facteurs) pour une évaluation significative des habiletés des acteurs impliqués dans le processus de détermination du statut de réfugié.

L'analyse des dossiers comprenait deux volets : 1 ) une analyse en profondeur de dix dossiers pour raffiner et compléter les facteurs définis, 2) une analyse complémentaire de trente autres dossiers.

Pour sélectionner les dix dossiers qui ont fait l'objet d'une analyse en profondeur, nous avons retenu plusieurs critères afin de constituer un échantillon varié. Nous avons tenu compte de pays d'origine comportant des risques pour la sécurité des personnes (Congo, Cameroun, Birmanie, Inde, Mexique, Kazakhstan, Rwanda, Honduras), du sexe des demandeurs, de leur âge, de leur orientation sexuelle et de leur situation de famille. Pour chaque analyse en profondeur, nous avons obtenu les cassettes et souvent les transcriptions des audiences, et nous les avons distribuées à l'une des trois équipes multidisciplinaires, chacune composée de trois analystes (juridique, psychologique et culturel).

Notre méthode analytique nous permet de faire des recommandations pour chaque catégorie d'acteurs du système, de même que des recommandations sur la structure et le fonctionnement du système. À notre connaissance, il s'agit de la première analyse multidisciplinaire en profondeur de la procédure de détermination du statut de réfugié.

\section{Résultats}

Nous présenterons en premier lieu quelques-uns des résultats décrivant l'état de la situation pour l'ensemble des dossiers considérés. Puis, nous exposerons les aspects saillants de l'analyse qualitative en utilisant des exemples précis pour illustrer les problèmes rencontrés dans chaque catégorie de facteurs.

Les résultats de l'analyse quantitative révèlent que seulement $20 \%$ des dossiers ne présentent qu'un type de problème (juridique, culturel ou psychologique), alors que $27,5 \%$ présentent des problèmes dans deux domaines et que $52,5 \%$ présentent des problèmes dans les trois champs étudiés. Ceci indique d'emblée un chevauchement très important entre les problèmes soulevés et peut permettre de comprendre pourquoi des efforts purement juridiques d'amélioration du système ont pu échouer.

En ce qui concerne les problèmes d'ordre juridique, ceux qui prévalent sont les problèmes d'administration de la preuve $(87,5 \%)$, la méconnaissance de certaines problématiques $(85 \%)$ et des conditions politiques du pays d'origine $(62,5 \%)$. Les problèmes d'interprétation du droit administratif et international sont également fréquents $(40 \%)$. Enfin, dans plus du quart des dossiers $(27,5 \%)$, les règles de conduite et de politesse ont été enfreintes.

Pour ce qui est des problèmes d'ordre psychologique, on relève des problèmes massifs d'évitement du matériel traumatique, manifestation habituelle du traumatisme transmis $(75 \%)$ et d'empathie (75\%). L'expression de préjugés $(67,5 \%)$ et les comportements pouvant dénoter un certain cynisme $(50 \%)$ sont également très présents. Enfin, dans plus du tiers des cas ( $35 \%)$, il est possible de déceler des signes de troubles émotionnels secondaires au traumatisme transmis chez les différents acteurs.

Dans le domaine culturel, la méconnaissance du contexte culturel, social et politique d'origine du réfugié s'avère un facteur principal $(72,5 \%)$. Une difficulté à apprécier justement les liens sociaux du requérant est également importante (52,5\%). Dans le cadre de l'audience et de la décision, les préjugés, les stéréotypes et les difficultés de communication jouent un rôle dans $42,5 \%$ des dossiers.

Pour illustrer ces résultats, nous présentons quelques exemples dans chaque catégorie de facteurs.

\section{Résultats juridiques}

Plusieurs acteurs, mais tout spécialement les commissaires et les agents d'audience, éprouvent des difficultés à évaluer la preuve.

- Les rapports d'experts et les témoignages d'experts : - Dans un cas, un rapport d'expert en psychologie a été produit en preuve, faisant état du syndrome de stress posttraumatique dont souffre le demandeur depuis qu'il a été torturé. Des photographies prises immédiatement après son arrivée au Canada ont été déposées en preuve, montrant son corps couvert de brûlures de cigarettes. Le psychologue avait conduit six entrevues consécutives du demandeur, faisant une évaluation complète de sa condition et établissant les liens avec son récit. La demande a été rejetée. Un des commissaires a dit, durant l'audience, qu'il prenait toujours les rap- 
ports d'experts " avec un grain de sel », et il a été découvert durant l'audience qu'il n'avait pas lu le rapport. La présidente a alors ajourné l'audience pour permettre à ce commissaire de lire le rapport. La présidente d'audience a également fait des remarques déplacées sur le fait qu'elle-même fumait, voulant sans doute dire qu'elle n'accordait pas beaucoup de poids aux marques de brûlures de cigarettes ou au rapport d'expert. Cela montre un important manque de compréhension de l'utilité et du poids de la preuve d'expert.

- Dans un autre cas, l'agent d'audience agit comme expert sur les fins d'un Thematic Apperception Test contenu dans un rapport d'expert («Ce n'est pas un test d'appréciation thématique, ce sont des images que le client doit apprécier de façon subjective. ») et fait par la suite des commentaires dédaigneux sur la subjectivité de la psychologie et en particulier de la psychanalyse. Ni les com-missaires ni l'agent d'audience ne font un examen sérieux du statut d'expert de la psychologue concernée, et ils déclarent globalement le rapport et le témoignage non crédibles, sans autre explication.

- Dans un cas du Kazakhstan, la requérante n'avait pas mentionné dans le Formulaire de ren-seignements personnels l'agression de son fils au cocktail Molotov. Durant l'audience, elle explique qu'elle ne voulait pas que l'on interroge l'enfant, dans le but de le protéger. Un rapport psychiatrique produit en preuve met en évidence les sentiments d'impuissance et de désarroi vécus par les parents lors de l'incident où leur fils a été brûlé par le cocktail Molotov: la mère, qui présentait également des symptômes traumatiques, a voulu épargner son fils de son mieux, en lui évitant une exposition à des stimuli pouvant rappeler le traumatisme. Les commissaires déterminent que la demande n'est pas crédible à cause de deux omissions, dont celle de ne pas avoir dévoilé l'agression du fils. Cette décision met en évidence une méconnaissance des mécanismes d'évitement qui se mettent en place après une situation traumatique, dans le cadre d'un désir maternel de protéger son enfant.

- Dans plusieurs cas, les commissaires montrent peu d'intérêt envers la preuve documentaire qui a été produite par l'avocat du demandeur, cette preuve n'étant souvent pas même mentionnée dans les motifs justifiant le rejet de la demande. Dans un cas, les commissaires ont affirmé dans la décision que la preuve do- cumentaire ne démontre aucunement une situation d'oppression à l'égard d'un groupe minoritaire auquel appartient le demandeur, ce qui contredit manifestement et sans explication le contenu des pièces déposées à l'audience.

- Plusieurs commissaires font un usage inadéquat de la connaissance d'office. Dans un cas, le président de l'audience a opposé sa connaissance d'office à la demanderesse relativement à la manière dont, dans les pays musulmans comme celui dont venait la demanderesse, lequel jouxte le pays de naissance du commissaire en question, les groupes d'étudiants qui voyagent à l'étranger, et particulièrement les filles, sont surveillés par des adultes : il conclut qu'il serait impossible que la demanderesse ait pu dormir chez la responsable du groupe hôte, alors que les parents de la demanderesse ne connaissaient pas cette responsable. Durant l'interrogatoire de la requérante par le président, l'avocate intervient pour lui demander des précisions sur la provenance de ces connaissances. Sur la bande enregistrée, cet échange entre le président et l'avocate est empreint d'un grand malaise. Le président interrompt l'avocate et ne lui laisse pas le temps de poser ses questions. Par peur d'avoir importuné le président, elle s'excuse à deux reprises auprès de ce dernier pour son « audace " pour finalement se taire. Pourtant, l'article 68(5) de la Loi sur l'immigration stipule qu'un commissaire doit non seulement informer le requérant de son intention d'admettre des faits d'office, mais également lui donner la possibilité de présenter ses observations. De plus, le président tente de faire une distinction inappropriée entre connaissance d'office « juridique » et connaissance d'office « culturelle».

- Les décisions de rejet sont souvent fondées sur le manque de crédibilité du demandeur. Ce manque de crédibilité est souvent fondé sur les contradictions entre les notes prises par le fonctionnaire d'immigration au point d'entrée au Canada, la Fiche de renseignements personnels que doit remplir le demandeur et son témoignage oral donné lors de l'audience. Dans plusieurs cas, ces « contradictions » sont minimes, ou ont été expliquées durant l'audience, ou auraient pu être expliquées si les commissaires avaient posé les questions appropriées.

- Dans un cas, la revendicatrice explique oralement que la personne chez qui elle habitait était l'une des dirigeantes d'un groupe culturel, mais précise que cette information manque à sa Fiche de rensei- 
gnements personnels, et que le problème proviendrait d'une confusion de la part de l'avocat ou d'une mauvaise traduction. Les commissaires refusent cette explication, parce qu'au début de l'audience la revendicatrice avait affirmé solennellement avoir eu une parfaite connaissance de sa Fiche de renseignements personnels, et que celleci lui fut traduite.

- Dans un cas de la Birmanie, l'interrogatoire de la requérante a duré cinq heures et a été effectué de manière désordonnée. On peut noter d'incessants retours sur des questions déjà posées et auxquelles la requérante a donné des réponses satisfaisantes. Ces retours ne sont pas justifiés par les commissaires et témoignent d'une attitude contradictoire qui frôle le harcèlement. De façon générale, ces retours créent une atmosphère tendue et laissent penser que les commissaires ne croient pas la requérante, sans qu'il soit possible de préciser quels sont les faits qui leur apparaissent non crédibles. De fait, la décision montre que les commissaires ne savent pas trop sur quel point attaquer la demande. Ce dossier dénote un manque de préparation préliminaire important qui se fait sentir tout au long de l'audience.

Les commissaires éprouvent des difficultés à conduire une audience :

- Dans un cas, la présidente a déclaré dès le début de l'audience qu'elle ne voulait pas entendre le récit du demandeur, puisqu' elle était d'avis que le demandeur raconterait la même histoire que celle qu' elle avait lue dans la Fiche de renseignements personnels. Elle rejette la requête de l'avocat qui voulait poser des questions de clarification au demandeur pour ensuite demander à l'agent d'audience de procéder à l'interrogatoire du demandeur et, finalement, rejeter la demande en raison d'un manque de crédibilité du récit. Ceci démontre une sérieuse méconnaissance du rôle d'une audience dans un processus décisionnel.

- Dans plusieurs cas, les commissaires et les agents d'audience débattent le sujet sans ménagement avec l'avocat ou le demandeur, ce qui dégénère parfois en de véritables altercations, indicatrices de conflits interpersonnels non maîtrisés. Dans d'autres cas, les commissaires montrent clairement qu'ils ne croient pas le récit, font des commentaires inadéquats ou montrent du cynisme. Tous ces cas indiquent des contraventions certaines au décorum et à l'étiquette desquels on attend le respect de la part de commissaires d'un tribunal administratif. Ces règles déontologiques existent pour que la justice soit rendue avec sérénité, en vertu du vieil adage selon lequel « la justice doit non seulement être rendue, mais il faut qu'il y ait apparence qu'elle a été rendue ».

Les motifs écrits des décisions montrent souvent des lacunes flagrantes, soit qu'ils contiennent seulement une liste de contradictions entre la Fiche de renseignements personnels et le témoignage oral, soit qu'ils soient simplement constitués d'une déclaration laconique disant que le demandeur n'est pas crédible, soit encore que les pièces déposées en preuve, et tout spécialement la preuve documentaire, n'y soient pas mentionnées.

- Dans un cas, les motifs débutent par une déclaration selon laquelle le témoignage du demandeur était très vague et manquait de précision, alors que l'écoute des enregistrements de l'audience révèle au contraire que le témoignage fut clair, articulé, précis, et que le demandeur a répondu aux questions calmement, précisément et complètement.

- Dans un cas du Congo, l'audience a porté sur des événements qui se sont déroulés après un viol qui est l'événement central pour fonder la demande de statut. La question du viol n'est pas abordée durant l'audience. La demande est rejetée sur des aspects mineurs de la demande. Les commissaires basent leurs décisions sur des contradictions entre la Fiche de renseignements personnels et le témoignage oral, comme la question de savoir quand exactement elle aurait pris conscience de la présence du beau-frère dans le véhicule qui a servi à son évasion. Cette " contradiction " suffit à elle seule, aux yeux des commissaires, non seulement pour rejeter la demande, mais pour conclure que cette demande n'a pas de minimum de fondement. Toute la question du viol par les militaires, $y$ compris l'existence d'un certificat médical l'attestant, n'a pas été soupesée par les commissaires, alors qu'il s'agissait d'éléments fondamentaux de la demande.

- Dans un cas de la Birmanie, la demanderesse n'a pas expliqué dans sa Fiche de renseignements personnels ce qu'elle a fait au Pakistan, où elle a demeuré six ans, car son avocat lui avait dit que cela n'était pas pertinent. Durant l'audience, les commissaires lui ont demandé d'expliquer pourquoi ce renseignement n'avait pas été mentionné dans sa fiche. L'avocat a répondu que cela n'avait rien à voir avec l'affaire. Néanmoins, la demanderesse a expliqué ce qu'elle avait fait au Pakistan. Dans la décision, les commissaires ont rejeté cette explication en déclarant que la demande- 
resse est une « personne instruite qui a la responsabilité de se décharger de son fardeau de preuve». En fait, les commissaires sous-entendent que son témoignage n'est pas crédible, car elle n'a pas dévoilé dans sa fiche tous les renseignements qu'ils estiment qu'elle aurait dû dévoiler.

- Les deux décisions où la demande est déclarée n'avoir pas de minimum de fondement sont très mal rédigées, alors qu'elles entraînent des conséquences désastreuses pour le demandeur qui ne peut plus exercer de recours judiciaires. Le raisonnement de ces dures décisions est faible et peu convaincant.

\section{Résultats psychologiques}

Tous les acteurs, mais tout spécialement les commissaires et les agents d'audience, ont des difficultés à transiger avec les effets de la transmission traumatiques.

- Réactions massives d'évitement : évitement direct, déni, normalisation de situations extrêmes (attitudes parfois cyniques).

- Dans un cas, la présidente d'audience répète à plusieurs reprises qu'elle ne veut pas entendre la description des tortures et du viol, et que la Fiche de renseignements personnels constitue une preuve suffisante sur ce point. Cependant, la demande est rejetée pour manque de crédibilité.

- Dans un autre cas, un jeune latino-américain homosexuel a été attaqué et violé par des soldats. Après avoir déposé une plainte, il fut cambriolé, menacé au téléphone et agressé de nouveau. Après son départ pour le Canada, son conjoint est assassiné avant de pouvoir le rejoindre. Dans son résumé oral des faits importants de la cause, l'agent d'audience omet de signaler le meurtre du conjoint, et ce fait essentiel n'a suscité à peu près aucune question.

- Dans un cas de Birmanie, les commissaires mettent l'emphase sur les renseignements factuels qui sont des détails de peu d'intérêt (détails géographiques, rôle de l'agent dans le renouvellement des passeports, etc.). Les commissaires passent rapidement sur l'arrestation, le viol et les mauvais traitements infligés à la requérante, les séquelles physiques et psychologiques (la honte, la peur, la façon de cacher ses blessures), le harcèlement et les insultes dus au racisme dans le pays d'origine et les conditions de survie difficiles dans un pays voisin. Après une seule question, ils changent rapidement de thème, abordant des informations plus factuelles (par exemple, le type de bâtiment où elle a été enfermée ou encore l'existence d'un permis pour manifester). Cela constitue non seulement un manque d'empathie de la part des commissaires, mais également une façon d'éviter ou de nier la souffrance de la requérante, en y accordant peu d'importance.

- Réactions émotives incontrôlées lorsque les acteurs sont confrontés aux expériences traumatiques (colère, victimes perçues comme agresseur, manque d'empathie, formation réactionnelle).

- Dans un cas, des militaires avaient attaqué deux hommes, laissant un d'entre eux en état de choc ou de coma. L'autre a demandé aux militaires agresseurs (seules personnes présentes) de l'aide pour son compagnon. Les militaires lui ont dit brutalement qu'ils s'en occupaient et qu'il devait quitter les lieux, ce qu'il a finalement fait : le corps du compagnon a été découvert quelques jours plus tard. Durant l'audience, un des commissaires a montré clairement qu'il ne croyait pas ce récit et a demandé avec colère, au revendicateur, à cinq minutes d'intervalle, d'abord comment il se faisait qu'il ait demandé de l'aide à ses tortionnaires, puis comment il se faisait qu'il ait pu laisser son compagnon entre les mains de ces derniers. Ces réactions montrent une réaction émotive très forte, un manque d'empathie et une association de la victime aux agresseurs, tous ces symptômes montrant une difficulté à transiger avec le fardeau psychologique engendré par le récit traumatique.

- Dans un cas de la Birmanie, le ton général est courtois au début de l'audience, mais monte progressivement. Les commissaires se montrent même parfois provocants, en reprenant les paroles de la requérante, en faisant preuve d'ironie, ou en riant entre eux. Par exemple, un commissaire demande à la requérante si elle est habituée à désobéir à ses parents. Ainsi, les commissaires semblent ainsi réaliser psychiquement une coupure par rapport à l'intensité émotive des problèmes vécus par la requérante, au point qu'ils ne sont pas disponibles pour une écoute empathique. En se protégeant psychiquement, ils se placent progressivement dans une position d'adversité, puis de mépris par rapport à la requérante, ce qui influe sur les réactions de celle-ci allant de l'anxiété (intonation, rythme d'élocution), à l'impatience (non-respect des règles de l'audience), puis à l'impuissance (pleurs). 
- Parfois, les conflits interpersonnels entre les acteurs (souvent l'avocat et les commissaires ou l'agent d'audience) se transforment en lutte ouverte pour le contrôle du déroulement de l'audience, au détriment de la décision à prendre qui devient l'enjeu de cette lutte.

Pour les avocats, une tendance à exposer leurs clients au risque d'une retraumatisation, afin de présenter un témoignage plus convaincant. Dans un cas, un avocat a accepté la suggestion de la présidente d'audience que le torse d'un enfant de onze ans soit dénudé durant l'audience de façon à montrer ses cicatrices, ce qui était totalement inutile puisqu'il y avait au dossier un rapport d'expertise médicale complet et des photos du corps de l'enfant. L'enfant a été sévèrement retraumatisé par cet incident, et les acteurs l'ont même noté au cours de l'audience.

Pour les professionnels de la santé :

- La tendance à prédire le comportement du demandeur devant le tribunal, en se fondant sur les entrevues thérapeutiques, alors que l'audition devant le tribunal est un environnement de survie radicalement différent de la relation sécurisante entre le patient et le professionnel. Si la prédiction ne se vérifie pas lors du témoignage, c'est tout le contenu de rapport d'expert qui peut être mis en cause au détriment de la crédibilité du demandeur.

- Le manque de clarté dans l'évaluation : l'évaluation de la capacité de témoigner n'est souvent pas distinguée de l'évaluation du récit du patient comme explication des symptômes.

\section{Résultats culturels}

Pour tous les acteurs, mais tout spécialement pour les commissaires et les agents d'audience, un manque de compréhension ou de sensibilité interculturelle qui se traduit souvent dans des manifestations claires de suspicion ou de cynisme envers le témoignage présenté par le demandeur, les témoins experts ou la preuve introduite par l'avocat. Ces malentendus culturels peuvent parfois conduire à des comportements agressifs de la part des commissaires, dans leur manière de rejeter la validité des témoignages ou de la preuve d'expert. Par exemple :

Une méconnaissance de la situation politique et sociale dans le pays d'origine du demandeur :

- Dans un cas du Rwanda, les commissaires montrent une méconnaissance flagrante et font des représentations simplistes de la situation génocidaire/postgénocidaire au Rwanda, des changements temporels et des transformations de la violence interethnique dans ce pays et des multiples formes de violence localisée entre les divers groupes, sous-groupes et milices. Par exemple, les commissaires estiment que la preuve montre que les différences entre les ethnies Hutu et Tutsi sont difficiles à établir, que les deux groupes sont également en danger, et que la violence actuelle est principalement fondée sur des considérations économiques. Pourtant, même s'il est vrai qu'il y a absence de marques distinctives entre ces deux groupes d'un point de vue strictement physique, le demandeur a clairement expliqué que dans les communautés, chacun sait en fait qui est Tutsi ou Hutu et que la persécution est basée sur cette connaissance.

- Dans un cas mexicain, la compréhension des commissaires du conflit au Chiapas est faible : ils semblent se le représenter comme un cas clair de guerre civile entre l'armée et des paysans sans terres révolutionnaires, expliquant qu'ils savent que les Indiens sont en lutte pour leurs terres depuis des décennies et que l'armée a été répressive, comme ils ont pu le lire dans les journaux. Ils prétendent ainsi qu'il est impossible que l'oncle du demandeur, un gradé de l'armée, ait pu acheter une terre au Chiapas en 1995, disant « Ça me semble complètement hors de contexte et inimaginable. » En fait, la plus grande partie du Chiapas est lourdement militarisée, malgré des poches de résistance du EZLN, et il n'est pas du tout inimaginable qu'un officier ait pu y acheter une terre.

- Dans un cas indien, la requérante explique l'absence de certificat médical mentionnant son viol. Elle invoque comme raison qu'en demandant cette preuve, son père aurait éprouvé une honte qui l'aurait forcée à courber la tête bien bas. Les commissaires n'apprécient pas cet argument à sa juste valeur et disent que la requérante a omis de présenter une preuve fondamentale que d'autres victimes originaires de cette région ont présenté spontanément pour passer l'examen d'acceptation à la cisR. On peut en déduire que les commissaires exigent que tous les demandeurs soient capables de transgresser les normes culturelles, quels que soient leur statut social ou familial, leur âge, l'influence paternelle et les tabous reliés à certaines valeurs.

Des représentations fausses de la vie quotidienne dans un pays en guerre ou en conflit, une présomption que toute normalité ou vie quotidienne est interrompue en temps de guerre et une perception de "contradiction » lorsque les récits des demandeurs ne correspondent pas à cette présomption. 
- Dans un cas du Kazakhstan, les commissaires se demandent comment la famille a pu continuer à vivre une vie relativement normale avant de finalement quitter le pays, c'est-à-dire en continuant de faire des affaires et en ne déménageant que lorsque la situation est devenue à ce point difficile, que quitter le pays était inévitable.

- Suivant une logique opposée, les commissaires demandent aux revendicateurs comment ils ont pu, en conscience, abandonner leur propre famille durant un temps de violence. Dans un cas mexicain, les commissaires mettent en doute la " désertion » de l'oncle du demandeur, après qu'il ait été visé par l'armée, tout comme le fait que le demandeur ne soit pas rentré chez lui après avoir subi des tortures pour s'enquérir de la situation de ses sœurs. L'idée ne semble pas effleurer les commissaires que, lorsqu'on a été étiqueté "subversif ", le maintien de contacts avec la famille peut mettre celle-ci en grave danger, particulièrement lorsque l'armée a pour stratégie de s'attaquer aux familles plus qu'aux individus, et que la seule option est de « disparaître ».

- Dans le cas du Kazakhstan, un manque de compréhension sur l'implantation locale de la violence est manifeste. Un des arguments centraux pour rejeter la demande est que les demandeurs n'ont pas été persécutés par les nationalistes kazakhs en raison de leur ethnie, mais avaient peur d'un individu en particulier. Dans la plupart des situations de violence ethnique, toutefois, les disputes locales préexistantes et la structure de pouvoir (entre individus, famille et groupe) sont interreliées dans la dynamique de la violence. Durant les périodes de querelles ethniques, alors que des populations particulières - les commissaires d'une ethnie particulière - sont la cible des autres, il arrive souvent que la différence ethnique soit utilisée par des personnes puissantes dans la communauté comme justification de la persécution, de l'oppression ou de la vengeance.

- Dans un cas rwandais, les commissaires déclarent que les Tutsis sont en sécurité au Rwanda, puisque le gouvernement rwandais est majoritairement composé de Tutsis et que ce gouvernement est démocratique, car il a relâché des prisonniers hutus. Ils rejettent le témoignage de l'expert qui décrit une situation beaucoup plus complexe et mouvante, parce qu'ils le jugent partial.

Des représentations simplistes de la cohérence culturelle (stéréotypes, etc.) :
- Dans un cas mexicain, les commissaires ne peuvent pas croire qu'une famille de classe moyenne au Chiapas n'a pas le téléphone, même si cela est commun dans cette région.

- Dans un autre cas d'Amérique latine, l'avocat présente des données et des articles montrant un nombre important de meurtres d'homosexuels. Les commissaires s'objectent à la pertinence de cette preuve en disant que les exemples portent sur des travestis et, dans l'intérêt de l'objectivité et de l'équité envers le demandeur, lui demandent s'il est un travesti, même si l'avocat explique que cette preuve révèle un climat généralisé de violence envers les homosexuels, les travestis étant particulièrement victimisés du fait de leur visibilité. Les commissaires déclarent que l'homophobie ne doit pas être si grave au Mexique, puisqu'un parlementaire est ouvertement gai et qu'il existe des communautés homosexuelles dans les grandes villes.

- Dans un cas rwandais, le commissaire ne peut comprendre comment un réfugié au Canada ne se souviendrait pas de l'adresse de son église à Montréal, alors que cela est fréquent parmi les réfugiés ou immigrants récents en provenance de pays dans lesquels l'orientation se fait par des marqueurs physiques et non par des adresses abstraites.

Malentendus culturels, insensibilité et préjugés fondés sur le sexe, l'origine ethnique, la religion et l'orientation sexuelle :

- Dans un cas impliquant un homosexuel, l'interprète a parlé de son partenaire en disant «este muchacho » ( « ce garçon »), ce qui est une façon condescendante de décrire une personne très importante pour le demandeur, personne qui fut assassinée à cause de lui. Dans le même cas, l'interprète interrompt le demandeur en lui disant de se tenir tranquille.

- Dans un cas indien, les commissaires considèrent comme étrange le comportement du père de la demanderesse : son attitude calme lorsqu'on lui annonce que sa fille a disparu (comme l'explique la demanderesse, il tentait de sauver sa réputation en tentant de faire croire que son absence n'était pas inconvenante) ou le fait qu'il ait encouragé sa fille à participer à un événement culturel et religieux dans une communauté voisine moins de un mois après son enlèvement et son viol (alors qu'il est clair, dans le contexte culturel et religieux de la famille, qu'il tentait de redonner à sa fille un certain équilibre). Ils ne paraissent pas comprendre à quel point un père de cette région doit 
protéger la réputation de sa fille et de sa famille, et quelle force de caractère cela requiert de cacher ainsi ses émotions aux étrangers.

- Dans un cas rwandais, les commissaires font une généralisation injustifiée lorsqu'ils affirment que, puisque le demandeur est un Adventiste et que les églises adventistes aident les réfugiés, il est étonnant qu'elles n'aient pas pu aider davantage le demandeur à quitter le pays.

\section{Résultats systémiques}

Même si l'objectif de notre recherche n'était pas de nature systémique, nous avons relevé et documenté des éléments dynamiques qui paraissent, au-delà des caractéristiques individuelles des acteurs, avoir une influence importante sur le processus.

Le caractère non contradictoire du processus semble créer beaucoup de confusion sur les rôles respectifs des acteurs. L'attitude interventionniste de nombreux commissaires crée souvent un climat tendu, fait de réactions émotives et parfois de comportements agressifs de la part de tous les acteurs. Cette situation a un impact négatif certain sur la sérénité de l'audience et ajoute à la confusion de nombreux revendicateurs qui ne savent plus qui ils doivent convaincre et contre qui ils doivent se défendre. En l'absence d'un "adversaire officiel ", l'avocat ne sait souvent pas quelle attitude adopter envers les commissaires, lesquels, souvent simultanément, se présentent comme les protecteurs des réfugiés et adoptent des attitudes agressives envers les revendicateurs. Si l'avocat se montre conciliant, il risque de paraître peu convaincant ou d'approuver des demandes inacceptables, comme le déshabillage du petit garçon en cours d'audience pour montrer ses cicatrices. S'il se montre agressif, il risque par contre de s'aliéner les commissaires.

Selon l'attitude des commissaires, les agents d'audience font souvent preuve de trop de prudence, posant des questions très générales ou proposant des conclusions inutiles. Dans un cas, l'agent d'audience s'est contenté d'affirmer que les commissaires devraient déterminer la crédibilité du revendicateur. Mais l'agent d'audience, surtout lorsque les commissaires interviennent peu, peut aussi se transformer en procureur public, procédant au contre-interrogatoire en règle du revendicateur, insistant sur chaque détail susceptible de révéler l'existence d'une " contradiction ». Dans bien des cas, l'agent d'audience est l'acteur le plus mal à l'aise dans le système : son rôle devrait être réévalué, quelles que soient les autres modifications adoptées.
Les interprètes eux-mêmes peuvent être affectés par des conflits interpersonnels non apparents entre les acteurs et peuvent avoir tendance à se ranger, pour des raisons émotives, du côté de l'un ou l'autre des acteurs, ce que leurs attitudes pourraient laisser paraître.

Ce climat général de tension se concrétise dans des conflits directs, ou il est déplacé à l'encontre d'un autre acteur.

De toute cette confusion résulte, pour plusieurs, un malaise dans lequel interagissent les acteurs. Non seulement y a-t-il confusion dans le rôle juridique de chaque acteur, mais en plus cette confusion a un impact énorme sur les dimensions psychologiques et culturelles, ces dernières ayant déjà été reconnues comme renforçant le sentiment généralisé d'incertitude.

\section{Recommandations}

Cette étude nous amène à trois séries de recommandations :

1. La sélection des commissaires et des agents d'audience doit être améliorée et fondée sur leurs compétences et leur expérience dans le domaine juridique, psychologique et culturel.

2. La formation de tous les acteurs doit être améliorée dans son ensemble et se poursuivre tout au long de leur carrière.

3. La nature inquisitoire du système pourrait être remise en question.

\section{Amélioration de la sélection des commissaires et des agents d'audience}

La fonction d'un commissaire est très souvent comparable à celle d'un juge, en ce sens que les conséquences de la décision (détention, mort, etc.) et les méthodes utilisées (audiences longues et émotives, preuve d'expert, etc.) sont similaires. On peut aussi trouver des similarités avec le travail d'une infirmière dans une unité de soins palliatifs, puisque tous deux ont à traiter constamment avec la douleur et la souffrance d'êtres humains et de leur famille. Écouter des histoires horribles sur une base quotidienne et prendre des décisions en se disant que toute erreur peut avoir des conséquences tragiques constituent de lourdes responsabilités professionnelles qui pèsent de tout leur poids dans l'équilibre psychologique de chacun. Dans les sessions de formation, certains commissaires expriment d'ailleurs parfois leur difficulté à assumer le poids des responsabilités de leur fonction et de leurs conséquences sur leur vie professionnelle.

1.1 Par conséquent, les commissaires devraient être sélectionnés (et devraient paraître avoir été sélection- 
nés) en fonction de leurs aptitudes et de leur expérience. Ces aptitudes et cette expérience devraient couvrir trois domaines :

- Connaissances juridiques : droit des réfugiés; droit de l'immigration; droits de la personne; conduite d'audiences; gestion des interactions avec les avocats et les revendicateurs; appréciation de la preuve; rédaction des décisions; etc.

- Expérience sur le terrain : travail dans des pays déchirés par la guerre ou par un conflit interne, travail au contact des réfugiés ou des personnes déplacées, sensibilité aux dynamiques particulières du travail avec des personnes issues d'autres cultures, en termes de communication et de compréhension; etc.

- Aptitudes psychologiques : capacité à supporter la souffrance de tous les acteurs, y compris la leur; expérience dans l'interaction avec des personnes traumatisées; etc.

Il ne serait pas nécessaire que les candidats retenus soient excellents dans ces trois domaines, mais il serait important qu'ils puissent faire preuve d'expérience et démontrer leurs habiletés dans les trois domaines, avec un certain degré d'excellence dans au moins un ou deux d'entre eux.

De cette manière, ils bénéficieront d'assez de confiance en eux-mêmes et de suffisamment de respect des autres acteurs du processus pour pouvoir utiliser, au besoin, leur position d'autorité afin d'imposer des standards de qualité dans la conduite de tous et chacun.

1.2 Compte tenu de l'importance de leur rôle dans la présente procédure, les agents d'audience devraient être sélectionnés, mutatis mutandis, en fonction de critères similaires à ceux des commissaires.

\section{Amélioration de la formation de tous les acteurs}

2.1 On devrait offrir à tous les acteurs un programme de formation permanent, bien conçu et cherchant à développer tant la sensibilité culturelle qu'un cadre conceptuel général de sciences sociales qui permette d'appréhender des enjeux comme la guerre, les inégalités et la détermination du statut de réfugié, les conséquences psychologiques de la violence organisée. Il ne faudrait pas que cette formation se limite à une simple énonciation des "différences » entre les autres normes culturelles ou modes de communication, ni qu'elle soit une simple liste des différentes crises politiques à travers le monde ou de leurs conséquences sur les personnes et les communautés. La formation devrait plutôt proposer une plus large discussion concernant 1 ) la construction, la perception et l'expérience de la différence culturelle; 2 ) les complexités et les nuances liées aux situations de violence politique, de conflits de faible intensité, de conflits ethniques, d'analphabétisme, de cultures rurales, etc.; 3) l'expérience vécue par les réfugiés.

2.2 Ce programme devrait inclure une formation continue en petits groupes, autour d'études de cas. Cela impliquerait une discussion autour d'anciens cas problématiques d'audiences et de décisions de la CISR, car il s'agirait d'analyser de façon critique ce qui est arrivé, et pourquoi, afin que les participants soient en mesure d'effectuer une remise en question de certaines hypothèses simplistes ou malencontreuses. La formation devrait mener à une réflexion sur la façon dont les trois dimensions, juridique, psychologique et culturelle, interagissent et ont un impact sur tous les acteurs du processus de détermination. Cette formation ne devrait pas être perçue comme une mise en cause de l'autorité des acteurs dans les cas discutés, mais comme une occasion offerte à ceux qui les étudient d'apprendre grâce aux expériences et aux erreurs des autres.

2.3 Une formation spéciale devrait être offerte à tous les acteurs sur l'utilisation et la valeur d'une expertise ou contre-expertise médicale, psychologique, culturelle ou politique, et sur le statut des experts.

2.4 Une formation spéciale devrait être offerte à tous les acteurs, ainsi qu'aux interprètes eux-mêmes, sur l'utilisation des interprètes davantage comme " médiateurs culturels » (cultural brokers) que comme simples traducteurs. Ils pourraient alors expliquer des situations, des histoires ou des expressions qui paraissent insensées au Canada, ce qui contribuerait à une meilleure interaction entre tous les acteurs dans la salle d'audience.

2.5 Une formation spéciale devrait être offerte à tous les acteurs sur un code de procédure et d'éthique dans le traitement des personnes victimes de torture ou sévèrement traumatisées, tel que celui développé par le Réseau d'intervention auprès des personnes ayant subi la violence organisée (RIVO), au nom du Réseau canadien pour la santé des survivants de la torture et de la violence organisée (ResCan).

2.6 Des groupes de soutien confidentiels devraient être constitués au sein de la CISR, afin de permettre aux 
commissaires et aux agents d'audience de relever, de partager et d'extérioriser les émotions et le stress reliés aux cas : peur, tristesse, impuissance, cynisme, etc. Ce support devrait aider les acteurs à gérer les effets de la transmission traumatique et à travailler dans une atmosphère plus sereine durant les audiences, ainsi qu'à faire preuve de plus d'empathie à l'égard des demandeurs de statut, quelle que soit la décision finale.

2.7 Des consultations individuelles avec une personneressource (psychologue ou autre professionnel compétent) devraient être offertes au sein de la CISR et même ouvertement encouragées, sans que puisse s'y attacher une connotation négative, puisque l'usage de telles consultations ne serait en aucun cas un signe de faiblesse ou d'incompétence, bien au contraire.

2.8 La CISR devrait collaborer avec des organisations professionnelles comme l'Association du Barreau canadien, afin d'offrir aux avocats une formation spéciale sur la façon d'éviter la retraumatisation de leurs clients, et sur la manière de reconnaître et de nommer les émotions présentes à l'intérieur de la salle d'audience, au lieu de les laisser dominer la scène.

2.9 La CISR devrait collaborer avec des institutions professionnelles, des organismes communautaires et des experts du domaine, afin de fournir aux médecins, aux psychologues et autres professionnels agissant à titre d'experts une formation sur la façon d'écrire leurs rapports d'expertise :

- dans un langage simple et accessible;

- dans une forme didactique, séparant clairement du reste les difficultés que le revendicateur pourrait avoir à affronter dans son explication des événements traumatiques qu'il a endurés;

- avec toute la sensibilité nécessaire en cas d'enjeux transculturels : ils devraient être en mesure (et formés au besoin) de nommer et d'expliquer toutes les situations dans lesquelles des différences de présentation, d'interprétation ou de réaction à des situations extrêmes, comparées avec celles des citoyens canadiens, pourraient affecter l'évaluation de la crédibilité du témoignage du revendicateur.

2.10 Dans toutes les modalités de formation proposées, une place importante devra être faite à la compréhension des enjeux du caractère non contradictoire de la procédure et du rôle de tous les acteurs dans un tel contexte.

\section{Remise en question de la nature inquisitoire du système}

3.1 Si les deux premières séries de recommandations ne sont pas mises en œuvre et si l'insatisfaction envers le système persiste, il sera probablement nécessaire de remettre en question la nature inquisitoire de la procédure.

3.2 Dans tous les cas, le rôle de l'agent d'audience dans un processus non contradictoire doit être clarifié.

\section{Conclusion}

Les résultats susmentionnés doivent être interprétés en tenant compte des limites de ce projet pilote : le caractère local des dossiers considérés, l'absence d'un calcul de fidélité interjuges lors de la codification, la taille relativement restreinte de l'échantillon. Ces limites invitent à la prudence quant à la possibilité de généraliser les résultats et suggèrent la nécessité d'études subséquentes. Celles-ci devraient idéalement se réaliser dans plusieurs lieux, compléter la perspective qualitative par un devis épidémiologique et permettre d'évaluer la prévalence des problèmes détectés. La participation directe de la CISR à la conception et à la réalisation d'une telle recherche serait un atout certain.

Dans le cadre de ces limites, nos résultats suggèrent cependant que des approches radicalement différentes sont nécessaires pour comprendre et résoudre les problèmes auxquels fait face la CISR. Il apparaît qu'une perspective multidisciplinaire est essentielle à la compréhension des facteurs personnels et systémiques qui ressortent comme problématiques : les lacunes soulevées montrent une interaction des aspects culturels, psychologiques et légaux des problèmes de perception et d'interprétation des différents acteurs qui ne peuvent être appréhendés dans une perspective purement administrative ou légale.

L'intégration des dimensions légale, culturelle et psychologique devrait non seulement être présente dans des recherches futures, mais également se refléter dans les critères de sélection et de formation continue des différents acteurs. Les commissaires, les agents d'audience et les autres professionnels associés au processus ont une lourde responsabilité d'assurer le droit d'asile et la protection des réfugiés au nom de la société canadienne. Seule une vision à la fois critique et créative du rôle de chacun et du système dans son ensemble peut nous permettre de mieux assumer ce mandat.

\section{Notes}

1. Les membres de la Commission de l'immigration et du statut de réfugié sont ici appelés " commissaires », et la Commission est désignée par son acronyme cisR. 
2. Crépeau, François, et France Houle. Compétence et Indépendance - Clefs de la crédibilité de l'Agence de protection, mémoire déposé auprès de la ministre de la Citoyenneté et de l'Immigration, 6 mars 1998, 34 p.

3. Bibeau, 1992.

4. Le travail des interprètes est délicat, puisqu'il s'agit de traduire non seulement les mots, mais aussi ce qu'ils signifient dans un contexte particulier (Jalbert, 1998). L'interprète est un intermédiaire qui peut faciliter la relation entre les autres acteurs ou, au contraire, générer des problèmes (Westermeyer, 1990). Pour la CISR, cela est particulièrement crucial lorsqu'il y a incompatibilité entre le demandeur et l'interprète pour des raisons de genre, d'appartenance sociale, religieuse ou ethnique.

5. L'expression des émotions en particulier est contenue dans certaines cultures, alors qu'elle peut être plus dramatisée dans d'autres. Le registre de ce qui peut être exprimé dépend aussi de l'interlocuteur et du contexte, amenant des variations qui peuvent paraître paradoxales voire contradictoires pour des observateurs informés.

6. Beiser, 1998.

7. Les commissaires sont soumis à des stress psychologiques importants, à cause, d'une part, de la teneur des histoires traumatiques qu'ils doivent écouter et, d'autre part, du poids psychologique des décisions qu'ils doivent rendre, lesquelles peuvent avoir des répercussions dramatiques sur la sécurité des demandeurs de statut. Les travaux sur les traumatismes de guerre après la Deuxième Guerre mondiale et la guerre du Vietnam ont mis en évidence que ceux-ci n'affectent pas uniquement ceux qui les vivent directement, mais aussi leurs proches et l'ensemble des réseaux sociaux auxquels ils appartiennent (Daniéli, 1998; Rousseau, 1998). La transmission indirecte des traumatismes, par un récit, a été particulièrement bien documentée chez les thérapeutes traitant des patients traumatisés. Dans la situation thérapeutique, c'est l'empathie des thérapeutes pour les victimes qui les rend particulièrement vulnérables, car ils ne peuvent plus utiliser les défenses habituelles d'évitement et de déni pour se protéger contre les représentations associées à l'histoire entendue.

8. Kleinman, 1997; Rousseau, 1992.

\section{Références}

Beiser, M., Dion, R., et A. Gotowiec. «Depression and Conduct Disorder in Native and Non-Native Children ", Journal of the American Academy of Child \& Adolescent Psychiatry, 37 (7 juillet), 1998, p. 736-742.

Bibeau, G., Chan-Yip, A. M., Lock, M., Rosseau, C., Sterlin, C., et H. Fleury. La santé mentale et ses visages: Un Québec pluriethnique au quotidien, Boucherville, Gaëtan Morin éditeur, 1992.

Danieli, Y. International Handbook of Multigenerational Legacies of Trauma, New York, Plenum Press, 1998.

Jalbert, M. « Travailler avec un interprète en consultation psychiatrique », Prisme, 8 (3), 1998, p. 94-112.

Kleinman, Arthur, et J. Kleinman. « The Appeal of Experience; The Dismay of Images: Cultural Appropriations of Suffering in Our Times ", Social Suffering. In V. D., Berkeley, University of California, Margaret Lock Ed., 1997, p. 1-24.
Rosseau, C., Dirié, M. S. T., Gagné, M. J., et G. Bibeau. «Between Myth and Madness, Dreaming the Departure. Premigratory Period for Young Somali Refugee ", Culture, Medicine and Psychiatry, 22, 1998, p. 385-411.

Rosseau, C., Drapeau, A., et E. Corin. Premigratory and Migratory Risk Factors for Refugee Children, présenté à l'Académie canadienne de pédopsychiatrie, Montréal, 1992.

Westermeyer, J. «Working with an Interpreter in Psychiatric Assessment and Treatment ", Journal of Mental and Nervous Disease, 178, 1990, p. 745-749.

Les auteurs remercient vivement :

- la coordinatrice principale du projet: $\mathrm{M}^{\mathrm{e}}$ Nathalie Blais;

- le personnel clé: Saliha Bahig Benkacem, Jocelyne Berthot, Catherine Gauvreau, Nicole Heusch, Denise Lainé, Anne-Charlotte Martineau, Delphine Nakache, Marian Shermarke;

- les collaborateurs et collaboratrices: Thérèse Aubut Gauvreau, Rivka Augenfeld, Gilles Bibeau, Geneviève Bourbonnais, $\mathrm{M}^{\mathrm{e}}$ Marie-France Bureau, Ayse Dalli, Janet Dench, Bawani Farzad, Nadia Ghalmi, Sujeta Gill, $\mathrm{M}^{e}$ Rosemary Hnatiuk, Carlos Hoyos, Louis Lafleur, Ginette Laforest, Myriam Lafrenière, Winnifred Langeard, $\mathrm{M}^{\mathrm{e}}$ Nathalie Lecoq, Gitanjali Lena, Angelica Marin-Lira, James Massé, Céline Procacci, Stephan Reichhold, Hélène Tessier, $\mathrm{M}^{e}$ Philippe Tremblay.

François Crépeau est Professeur titulaire au Département des sciences juridiques de l'uqam, où il enseigne le droit constitutionnel et le droit international. Il est le Directeur fondateur du Centre d'études sur le droit international et la mondialisation (cedim) et Directeur de la Revue québécoise de droit international.

Patricia Foxen, M.P.H. (Columbia), M.A. (McGill) est étudiante de $3^{e}$ cycle en Anthropologie à l'Université McGill. Elle fait présentement de la recherche au Service de psychiatrie transculturelle à l'Hôpital de Montréal pour Enfants.

Cécile Rousseau est Professeur-associé de Psychiatrie à l'Université McGill et est membre d'ERASME (Équipe de recherche et d'action en santé mentale et culture). Elle dirige l'équipe de Psychiatrie pédiatrique transculturelle à l'Hôpital de Montréal pour Enfants.

France Houle enseigne le Droit administratif à l'Université de Montréal. Son domaine de recherche concerne les preuves et les procédures devant les tribunaux administratifs et l'analyse des aspects théoriques liés à diverses méthodes utilisées par l'administration pour formuler des règlements. 